\title{
A spatio-temporal mathematical model for the dynamics of paclitaxel in cell monolayers.
}

Isaac Proudfoot ${ }^{1}$, Giulia Vallardi ${ }^{2}$, Yoko Shishikura ${ }^{2}$, Ola Epemolu ${ }^{2}$, Adrian T. Saurin ${ }^{3}$, Mariya Ptashnyk ${ }^{4}$, and Philip J. Murray $^{1}$

${ }^{1}$ Department of Mathematics, University of Dundee, Dundee, DD1 $4 \mathrm{HN}$

${ }^{2}$ School of Life Sciences, University of Dundee, Dundee, DD1 5EH

${ }^{3}$ Division of Cellular Medicine, School of Medicine, University of Dundee, Dundee, DD1 9SY

${ }^{4}$ School of Mathematical and Computer Sciences, Heriott-Watt University, Edinburgh, EH14 4AS

\begin{abstract}
The authors have withdrawn their manuscript due to the discovery of fundamental issues with the implementation of the ODE/PDE model, whose output was shown in Figure 5 of the original version of this paper, rendering the results and conclusion unreliable. Therefore, the authors do not wish this work to be cited as reference for the project. If you have any questions, please contact the corresponding author.
\end{abstract}

